\title{
Resource-effective serosurveillance for the detection of West Nile Virus in Switzerland using abattoir samples of free-range laying hens
}

\author{
Vogler, Barbara Renate ; Hartnack, Sonja ; Ziegler, Ute ; Lelli, Davide ; Vögtlin, Andrea ; Hoop, Richard ; Albini,
} Sarah

\begin{abstract}
West Nile virus (WNV) is an important zoonotic pathogen maintained in a natural transmission cycle between mosquitoes and birds as reservoir hosts. In dead-end hosts, such as humans, infection may result in fatal neurologic disease translating into disease and death-related suffering and increased health care costs. In humans, WNV may also be transmitted through blood transfusions and organ transplants. WNV is not present in Switzerland yet, but competent vector species (especially Culex pipiens and Aedes japonicus) are prevalent and an introduction of the virus, likely through wild birds, is expected at any time. Therefore, it is important for Switzerland to be prepared and establish a surveillance system for WNV to initiate increased prevention activities, such as the screening of blood and organ donations and public education activities in case virus circulation is detected. The long-term goal of these surveillance measures would be a reduced infection rate in humans resulting in less suffering and reduced health care costs. To provide the basis for a pragmatic and resourceeffective WNV surveillance program, this study used aliquots of serum samples of free-range laying hens taken at the abattoir and collected in the frame of the ongoing Swiss Avian Influenza and Newcastle Disease monitoring program for a 2-year period. All 961 aliquots were analyzed using a commercial competitive WNV enzyme-linked immunosorbent assay (ELISA). The study allowed to set up sampling and laboratory routines as a basis for future WNV surveillance activities. At this stage there is no evidence for circulation of WNV in Switzerland.
\end{abstract}

DOI: https://doi.org/10.1089/vbz.2018.2319

Posted at the Zurich Open Repository and Archive, University of Zurich

ZORA URL: https://doi.org/10.5167/uzh-168751

Journal Article

Accepted Version

Originally published at:

Vogler, Barbara Renate; Hartnack, Sonja; Ziegler, Ute; Lelli, Davide; Vögtlin, Andrea; Hoop, Richard; Albini, Sarah (2019). Resource-effective serosurveillance for the detection of West Nile Virus in Switzerland using abattoir samples of free-range laying hens. Vector Borne and Zoonotic Diseases, 19(3):222-224.

DOI: https://doi.org/10.1089/vbz.2018.2319 


\title{
Resource-Effective Serosurveillance for the Detection of West Nile Virus in Switzerland Using Abattoir Samples of Free-Range Laying Hens
}

\author{
Barbara R. Vogler, ${ }^{1}$ Sonja Hartnack, ${ }^{2}$ Ute Ziegler, ${ }^{3}$ Davide Lelli, ${ }^{4}$ Andrea Vögtlin, \\ Richard Hoop, and Sarah Albini ${ }^{1}$
}

\begin{abstract}
West Nile virus (WNV) is an important zoonotic pathogen maintained in a natural transmission cycle between mosquitoes and birds as reservoir hosts. In dead-end hosts, such as humans, infection may result in fatal neurologic disease translating into disease and death-related suffering and increased health care costs. In humans, WNV may also be transmitted through blood transfusions and organ transplants. WNV is not present in Switzerland yet, but competent vector species (especially Culex pipiens and Aedes japonicus) are prevalent and an introduction of the virus, likely through wild birds, is expected at any time. Therefore, it is important for Switzerland to be prepared and establish a surveillance system for WNV to initiate increased prevention activities, such as the screening of blood and organ donations and public education activities in case virus circulation is detected. The long-term goal of these surveillance measures would be a reduced infection rate in humans resulting in less suffering and reduced health care costs. To provide the basis for a pragmatic and resource-effective WNV surveillance program, this study used aliquots of serum samples of free-range laying hens taken at the abattoir and collected in the frame of the ongoing Swiss Avian Influenza and Newcastle Disease monitoring program for a 2-year period. All 961 aliquots were analyzed using a commercial competitive WNV enzyme-linked immunosorbent assay (ELISA). The study allowed to set up sampling and laboratory routines as a basis for future WNV surveillance activities. At this stage there is no evidence for circulation of WNV in Switzerland.
\end{abstract}

Keywords: West Nile virus, surveillance, Switzerland, competitive ELISA, sentinel chickens

\section{Introduction}

W EST NILE VIRUS (WNV) is a flavivirus belonging to the Japanese encephalitis virus (JEV) group, maintained in a transmission cycle between mosquitoes and birds. Birds are usually asymptomatic carriers. Free-range chickens can be used as sentinels to detect early circulation of WNV through seroconversion (Buckley et al. 2006, Rizzoli et al. 2007). Infection of incidental hosts, such as humans, may result in febrile illness to sometimes fatal meningoencephalitis. Transmission between humans may occur vertically (transplacental or through nursing) or horizontally (through blood or organ donations) (WHO 2011).

WNV is prevalent in parts of southern Europe with several affected countries neighboring Switzerland, that is, Italy, France, and Austria (Rizzo et al. 2016, Gossner et al. 2017). In Switzerland, competent vector species, including Culex pipiens, the principal vector of WNV (Engler et al. 2013), and the invasive Aedes japonicus (Veronesi et al. 2018) are widely prevalent with locally high abundances (Wagner et al. 2018), and an introduction of the virus, likely through wild birds, is expected at any time.

\footnotetext{
${ }^{1}$ Vetsuisse Faculty, Institute of Veterinary Bacteriology, National Reference Centre for Poultry and Rabbit Diseases (NRGK), University of Zurich, Zurich, Switzerland.

${ }^{2}$ Section of Epidemiology, Vetsuisse Faculty, University of Zurich, Zurich, Switzerland.

${ }^{3}$ Friedrich-Loeffler-Institut, Federal Research Institute for Animal Health, Institute of Novel and Emerging Infectious Diseases, Greifswald-Insel Riems, Germany.

${ }^{4}$ Istituto Zooprofilattico Sperimentale della Lombardia e dell'Emilia Romagna (IZSLER), Brescia, Italy.

${ }^{5}$ Institute of Virology and Immunology, Bern \& Mittelhäusern, Switzerland.

${ }^{6}$ Department of Infectious Diseases and Pathobiology, Vetsuisse Faculty, University of Bern, Bern, Switzerland.
} 
When WNV disease was classified as notifiable in 2011, the Swiss government suggested to establish the appropriate diagnostic methods for detection of virus and antibodies at the Swiss reference centers for WNV. This would provide a basis for rapid installation of a structured surveillance program for WNV (in birds), once it is introduced to Switzerland. The first yearly detection may then trigger increased prevention activities, such as the screening of blood and organ donations and public education activities. The long-term goal of these surveillance measures would be a reduced infection rate in humans resulting in less suffering and reduced health care costs.

This study tested the validity and established the laboratory use conditions of a commercial enzyme-linked immunosorbent assay (ELISA), a frequently used method to detect the concentration of antibodies in blood serum, at the National Reference Centre for Rabbit and Poultry Diseases (NRGK) to detect anti-WNV antibodies in chicken.

It was decided that the samples to test for seroconversion had to fulfill the following criteria: they originated from chicken that had been exposed to WNV vectors during the WNV transmission season (May to October) and were old enough to have developed an immune response during that period. In addition, the samples had to be geographically as randomly distributed as possible and-considering that the described approach may be used as a basis for a future surveillance program - that the samples were current.

All listed criteria were fulfilled by the samples taken from free-range laying hens collected at the abattoir in the frame of the national Avian Influenza and Newcastle Disease monitoring program (Swiss AI/ND-Monitoring; FSVO 2017). Therefore, this study used aliquots of these samples with the aim to design a pragmatic and resource-effective WNV surveillance program that may be readily extended once WNV is detected in Switzerland for the first time.

\section{Materials and Methods}

A total of 961 aliquots of free-range laying hen abattoir serum samples were obtained from Swiss AI/ND-Monitoring between February 2014 and November 2016. The samples originated from 98 randomly selected flocks. All sampled animals had appeared healthy.

In addition, 18 negative sera from specific pathogen-free chickens, 28 positive sera of 25 field-infected horses, and 3 experimentally infected chickens were used for validation purposes. To further confirm the trustworthiness of test results, 15 serum samples provided by ANSES (laboratory proficiency test by Laboratoire de Santé Animale de Maisons-Alfort) were used for internal validation.

All serum samples were tested with the "ID Screen ${ }^{\circledR}$ West Nile Competition Multi-species" ELISA (ID Vet, France) according to the manufacturer's protocol and measured on a Multiskan ${ }^{\circledR}$ Ex (Thermo Fisher Scientific, Waltham, MA). Sample results were categorized as negative, doubtful, and positive as instructed in the protocol. As cross-reactions with antibodies to closely related viruses from the JEV group are possible, samples with doubtful or positive results were sent to the National Reference Laboratory for WNV in Germany (Federal Research Institute for Animal Health, FLI) for confirmation. Negative results were considered as trustworthy.

\section{Results}

All 961 samples were analyzed for anti-WNV antibodies, with 959 samples being tested negative. One doubtful and one positive sample underwent a rigorous retesting at the FLI. The same ELISA kit showed negative results and a virus neutralization test did not detect neutralizing antibodies against WNV, JEV, Usutu virus (USUV), or tick-borne encephalitis virus (TBEV). The samples were, therefore, considered as "false positive" test result by the first ELISA.

The additional negative and positive sera were all tested clearly negative and positive, respectively. The participation in the proficiency test showed satisfactory results. Unsurprisingly, the three samples of the test panel containing antibodies against USUV, TBEV, and JEV, respectively, were detected as positive as well.

\section{Discussion}

A commercial competitive ELISA was successfully tested and established at the NRGK using Swiss free-range laying hen samples. A total of 961 samples tested negative for WNV antibodies, suggesting that WNV is currently not circulating in Switzerland. According to the formula given by Thrusfield (2005) for an approximate annual number of 2.43 million laying hens with access to an outdoor climate area in Switzerland (Federal Office for Agriculture, FOAG 2016) with the number of samples tested negative and an assumed minimal prevalence of $5 \%$, the maximum number of nondetected positive free-range laying hens in Switzerland would be 22,688 . Therefore, geographically restricted smallscale circulation between wild bird reservoir species cannot be strictly excluded. An ongoing targeted study of the NRGK to detect WNV RNA in Swiss wild birds might provide further insights.

Only 2 of 961 samples initially gave false positive ELISA results, and as expected, the cross-reactivity of the ID Vet ELISA test with antibodies of other flaviviruses of the JEV group, including USUV, as stated by the manufacturer, was confirmed by the results of the proficiency test. As sentinel chickens are suggested to be well suited for surveillance purposes of flaviviruses, including USUV (Lelli et al. 2008), the lack of USUV detection using a cross-reacting ELISA kit suggests that there was no active circulation of USUV in Switzerland during the study period (Vazquez et al. 2011). This is surprising, as repeated outbreaks have been documented for Switzerland (FSVO 2016) and different authors (e.g., Savini et al. 2011, Weissenböck et al. 2013) expect a silent circulation of USUV for European countries with occasional outbreaks. However, little is known about the epidemiology of USUV and up to now, it was not described whether the virus is geographically evenly distributed or if it occurs in pockets of high prevalence.

In conclusion, the commercial WNV ELISA is robust, but positive ELISA reactions need to be reconfirmed by flavivirus-specific neutralization assays.

The synergistic approach of this study shows that passively acquired samples with no need to pay for extra manpower in the field is both cost-saving and adequate. The established sampling and laboratory routines can be used as a basis for the creation of a structured surveillance program once it is deemed necessary to be implemented. In addition, the sample size-and, therefore, sensitivity of surveillance-may be 
rapidly and easily increased by including suitable aliquots of serum samples of free-range chicken sent to the NRGK for herd health monitoring.

This study will be ongoing, trusting that the used sampling and analysis methods are sufficient to timely detect an establishment of WNV in Switzerland. We propose, however, that after first detection of WNV, surveillance activities are intensified and adjusted to the current situation to early detect seasonal WNV circulation.

\section{Acknowledgement}

The authors thank Antonio Lavazza for carefully reading the article.

\section{Author Disclosure Statement}

No competing financial interests exist.

\section{References}

Buckley A, Dawson A, Gould E. Detection of seroconversion to West Nile virus, Usutu virus and Sindbis virus in UK sentinel chickens. Virol J 2006; 3:1-6.

Engler O, Savini G, Papa A, Figuerola J, et al. European Surveillance for West Nile virus in mosquito populations. Int $\mathbf{J}$ Environ Res Public Health 2013; 10:4869-4895.

FOAG. Agrarbericht 2016. 2016. Available at https://www .agrarbericht.ch/de/politik/direktzahlungen/produktionssysteme

FSVO. Bericht zur Überwachung von Tierseuchen Daten 2016. 2017. Available at https://www.blv.admin.ch/blv/de/home/ tiere/tiergesundheit/ueberwachung/nationales-ueberwachungs programm.html

Lelli R, Savini G, Teodori L, Filipponi G, et al. Serological evidence of USUTU virus occurrence in North-Eastern Italy. Zoonoses Public Health 2008; 55:361-367.

Gossner C, Marrama L, Carson M, Allerberger F, et al. West Nile virus surveillance in Europe: Moving towards an integrated animal-human-vector approach. French Agency for Food, Environmental and Occupational Health \& Safety (ANSES) 2017; 22:1-10.
Rizzo C, Napoli C, Venturi G, Pupella S, et al. West Nile virus transmission: Results from the integrated surveillance system in Italy, 2008 to 2015. Euro Surveill 2016; 21:1-8.

Rizzoli A, Rosa R, Rosso F, Buckley A, et al. West Nile virus circulation detected in northern Italy in sentinel chickens. Vector-Borne Zoonot 2007; 7:411-417.

Savini G, Monaco F, Terregino C, Di Gennaro A, et al. Usutu virus in ITALY: An emergence or a silent infection? Vet Microbiol 2011; 151:264-274.

Thrusfield M. Veterinary Epidemiology. Oxford: Blackwell Science Ltd., 2005.

Vazquez A, Jiménez-Clavero MA, Franco L, Donoso-Mantke O, et al. Usutu virus-Potential risk of human disease in Europe. Euro Surveill 2011; 16:19935.

Veronesi E, Paslaru A, Silaghi C, Tobler K, et al. Experimental evaluation of infection, dissemination, and transmission rates for two West Nile virus strains in European Aedes japonicus under a fluctuating temperature regime. Parasitol Res 2018; 117:1925-1932.

Wagner S, Guidi V, Torgerson PR, Mathis A, et al. Diversity and seasonal abundances of mosquitoes at potential arboviral transmission sites in two different climate zones in Switzerland. Med Vet Entomol 2018; 32:175-185.

Weissenböck H, Bakonyi T, Rossi G, Mani P, et al. Usutu Virus, Italy, 1996. Emerg Infect Diseases 2013; 19:1996-1999.

WHO. West Nile virus-Fact sheet $N^{\circ} 354$, July 2011. 2011. Available at www.who.int/mediacentre/factsheets/fs354/en

Address correspondence to:

Barbara R. Vogler

Vetsuisse Faculty

Department of Poultry and Rabbit Diseases Institute of Veterinary Bacteriology University of Zurich

Winterthurerstr. 270

8053 Zürich

Switzerland

E-mail: bvogler@vetbakt.uzh.ch 